\title{
Frequency Estimation of Single-Tone Sinusoids Under Additive and Phase Noise
}

\author{
Asmaa Nazar Almoosawy \\ MSc Candidate \\ Department of Physics \\ Faculty of Education for Women \\ University of Kufa, Najaf, Iraq
}

\author{
Zahir M. Hussain \\ Professor; Dept. of Computer Science \\ University of Kufa \\ P.O.Box 21, Kufa, Najaf, Iraq \\ Professor (Adjunct), ECU, Australia
}

\author{
Fadel A. Murad \\ Assistant Professor \\ Department of Physics \\ Faculty of Education for Women \\ University of Kufa, Najaf, Iraq
}

\begin{abstract}
We investigate the performance of main frequency estimation methods for a single-component complex sinusoid under complex additive white Gaussian noise (AWGN) as well as phase noise (PN). Two methods are under test: Maximum Likelihood (ML) method using Fast Fourier Transform (FFT), and the autocorrelation method (Corr). Simulation results showed that FFT-method has superior performance as compared to the Corr-method in the presence of additive white Gaussian noise (affecting the amplitude) and phase noise, with almost $20 \mathrm{~dB}$ difference.
\end{abstract}

Keywords-Frequency Estimation; Correlation; Cramer-Rao Bound; Phase Noise; Maximum Likelihood Estimator

\section{INTRODUCTION}

The frequency estimation (IF) of a complex sinusoidal signal in white Gaussian noise is one of the major problems in the literature. This is so because IF has been applied widely in many areas such as radar, sonar, communications and image analysis [1-5]. There is a variety of approaches to the frequency and phase estimation problem, with differences in performance as regards frequency estimation accuracy and computational complexity [5]. In many applications, it is necessary to detect the frequency of a single tone in a noisy environment. Taking the Discrete Fourier Transform (DFT) using FFT algorithm of the collected samples is the most common method of making such a frequency estimate. Practical limitations like the computational complexity can restrict the number of samples under processing (hence, the amount of signal information), a factor that will restrict the resolution of the estimate provided by the DFT [6]. The maximum likelihood estimator (MLE) to estimate the frequency of a sinusoid damaged by additive Gaussian noise was thoroughly studied by Rife and Boorstyn [7]. Quinn [8] developed a simple and efficient method to estimate the frequency of a single-tone sinusoidal signal based on the three samples around the DFT maximum (peak). A similar method was developed by Grandke [9]; this method uses the DFT maximum point (in the frequency domain) along with only one adjacent frequency. Both of the above methods are efficient in frequency estimation in terms of good performance (accuracy of frequency estimation) at higher noise powers (i.e., low SNRs that may reach $0 \mathrm{~dB}$ ). However, neither of these two methods can directly give a good magnitude estimate, also, both methods require division operation [6].
In this paper we will estimate the frequency of a single-tone sinusoid under AWGN and phase noise (PN) using two most popular methods: MLE method through using Fourier Transform (FT) (calculated by Fast Fourier Transform algorithm, FFT), and the Correlation method (Corr). The latter has been traditionally preferred to MLE for being computationally less intensive than FFT. Frequency estimation based on Fourier transformation is explained in Section 2, while in Section 3 we explain the autocorrelation method. Section 4 provides simulation results and performance comparison between the two methods.

\section{FREQUENCY ESTIMATION BASED ON FOURIER TRANSFORM}

Let the signal to be a single-tone sinusoid as follows:

$x(t)=A \cdot \sin \left(\omega_{0} t+\emptyset_{0}\right)+\epsilon(\mathrm{t})$

where, $A$ is the signal amplitude, $\omega_{0}$ is the frequency of the signal, $\emptyset_{\mathrm{o}}$ is the initial phase and $\epsilon(\mathrm{t})$ is an additive noise process. Noise is assumed to be Gaussian white noise process with $\varepsilon[\epsilon]=0$ ( $\mathcal{E}$ being the expectation functional) and var $[\epsilon]=$ $\sigma^{2}$.

Assuming that all the above parameters are unknown, we try to get an estimate for the frequency $\omega_{0}$ as $\widehat{\omega}$. The estimate should be as accurate as possible, also, it should not be computationally intensive [10].

Two important quantities associated with any estimate is the bias, $b[\widehat{\omega}]=\mathcal{E}[\widehat{\omega}]-\omega$, and the variance, given by $\operatorname{var}[\widehat{\omega}]=\varepsilon\left[(\widehat{\omega}-b(\widehat{\omega}))^{2}\right]$.

For unbiased estimators (bias $=0$ ), an important performance measure is the Cramér-Rao bound (CRLB), which represents the minimum possible variance for the unbiased estimator when noise effect decreases or the Signal-to-Noise Ratio (SNR) increases. The CRLB of the unbiased frequency estimator has been formulated as follows [11]:

$\mathrm{CRB}_{\omega}=\frac{1}{\operatorname{SNR}} \frac{6}{N\left(N^{2}-1\right)}$

where $N$ is the number of signal samples and SNR is the signal - to - noise ratio $\left(=A^{2} /\left(2 \sigma^{2}\right)\right)$.

We know that FT method estimates the frequency by the peak of the Fourier Spectrum $X(f)$ of the sinusoidal signal 
$x(t)$, computed from the sampled signal $x(n)$ by the DFT as $X(k)=\frac{1}{\sqrt{N}} \sum_{n=0}^{N-1} x(n) \exp \left(-\frac{2 \pi n k}{N}\right)$.

However, the actual frequency of a signal may not fall on one of the above frequencies of the DFT bins, hence; we use the magnitudes of the nearby bins to determine the actual signal frequency through the process of interpolation. There are several interpolation methods as follows.

\section{A. Quadratic Interpolation:}

This method finds a quadratic fit $y=a+b x+c x^{2}$ in the neighborhood of the maximum $\max \{X(f)\}$ with the three points [5]:

$\left(K-1, u_{1}=\left|X_{K-1}\right|\right)$,

$\left(K, u_{2}=\left|X_{K}\right|\right)$,

and $\left(K+1, u_{3}=\left|X_{K+1}\right|\right)$,

where $K=\arg \left\{\max _{k}[X(k)]\right\}=$ index of the absolute maximum magnitude of the DFT, which refers to the actual frequency $F=K f_{s} / N, f_{s}$ being the sampling frequency.

Now the actual maximum given by the quadratic formula above will be at the point $y=-b /(2 c)$ as follows:

$u=K+d$;

where $d=\left(u_{3}-u_{1}\right) /\left[2 *\left(2 * u_{2}-u_{1}-u_{3}\right)\right]$.

The estimated frequency is $F_{o}=\frac{u f_{s}}{N}$.

The Barycentric method is similar, with $u=K+d$; where $d=\left(u_{3}-u_{1}\right) /\left(u_{1}+u_{2}+u_{3}\right)$.

\section{B. Quinn's First Estimator [8]:}

Taking the three DFT points:

$\left(K-1, v_{1}=X_{K-1}=r_{1}+i \cdot s_{1}\right)$,

$\left(K, v_{2}=X_{K}=r_{2}+i \cdot s_{2}\right)$,

and $\left(K+1, v_{3}=X_{K+1}=r_{3}+i \cdot s_{3}\right)$,

we perform the following calculations:

$R=r_{2}^{2}+s_{2}^{2}$

$p=\left(r_{3} \cdot r_{2}+s_{3} \cdot s_{2}\right) / R$

$g=-p /(1.0-p)$;

$q=\left(r_{1} \cdot r_{2}+s_{1} \cdot s_{2}\right) / R$;

$e=q /(1.0-q)$;

If $(p>0)$ and $(q>0)$ then, $d=p$, else, $d=q$,

Now: $u=K+d$.

\section{Quinn's Second Estimator [12]:}

Using the above three points with other quantities, we have:

$d=\frac{p+q}{2}+h\left(p^{2}\right)+h\left(q^{2}\right) ; \quad u=K+d$,

where $h(x)=\frac{\frac{1}{4} \ln \left(3 x^{2}+6 x+1\right)-\frac{\sqrt{6}}{24} \ln \left(x+1-\sqrt{\frac{2}{3}}\right)}{x+1+\sqrt{\frac{2}{3}}}$.

Estimating the frequency $f_{o}=\omega_{o} / 2 \pi$ using Quinn's second estimator has the least RMS error; however, in our simulation we used the Quadratic Method with frequency compensation:

$d=\frac{\left(u_{3}-u_{1}\right)}{\left(4 * u_{2}-2 u_{1}-2 u_{3}\right)}$

\section{FREQUENCY ESTIMATION BASED ON AUTOCORRELATION}

The autocorrelation algorithms are to extract the frequency from the phase of the available signal's autocorrelation with fixed lags.

The periodogram-based estimators use the Discrete Fourier Transform (DFT) for a coarse search and an interpolation technique for a fine search [13]. In correlation-based singletone frequency estimation, consider the single-tone model as per Equation (1). For correlation-based estimators, an estimate of the frequency is obtained by the information of one or several estimated entries of the auto-correlation sequence of $x(n)$ :

$r(m)=\mathcal{E}\left[x(n) x^{*}(n-m)\right]=|A|^{2} e^{i 2 \pi f_{o} m}+\sigma^{2} \delta_{m, 0}$

where $(\mathcal{E}[\cdot])$ denotes statistical expectation, $\delta_{m, 0}$ is the Kronecker delta, $\sigma^{2}$ is the noise variance as defined in Equation (1), and (*) denotes complex conjugation. Note that since noise is uncorrelated with itself, its autocorrelation is a delta function (exists at lag $m=0$ only).

We can find the autocorrelation sequence $\{r(m)\}$ from the data sequence as follows:

$r(m)=\frac{1}{N-m} \sum_{n=0}^{N-1} x(n) x^{*}(n-m)$

Note that $r(m)=r(-m)$.

From Equation (5), we may have close information about the frequency $f_{o}$ from the phase angle of $\{r(m)\}$, that is, if we exclude the case $m=0$ in order not to interfere with the noise effect, we have:

$m \cdot \omega_{o}=$ phase $[r(m)]\langle\bmod [2 \pi]\rangle$

$m \cdot \omega_{o}=$ phase $[r(m)]+2 \pi k \quad ; k=$ integer

The integer $k$ satisfies $0 \leq k<m$. As we want positive results for frequency, the angle and $\bmod [2 \pi]$ operation are restricted to the interval $[0,2 \pi)$. Also, only positive values of $m$ are considered in our simulations.

The first possible frequency estimate from Equation (7) is obtained by putting $k=0$; hence, if we choose the first autocorrelation sample at $m=1$, we have:

$\omega_{o}=$ phase $[r(1)]$

This estimator is known as the minimal order linear predictor [14]. It is also a special case of the Pisarenko harmonic decomposer frequency estimator [15]. It is shown that the performance of this linear predictor can be improved by using a different correlation lag [16]. In [17] - [18], it was shown that the estimator based on a single correlation coefficient can be made more efficient.

A disadvantage with the above estimators (other than the fundamental estimator) is the ambiguity to the frequency estimate [19], [20]. It is shown in [21] that the frequency ambiguity could be resolved using two correlations with relatively prime correlation lags; this is further explained in [22], [23]. 


\section{FREQUENCY ESTIMATION UNDER GAUSSIAN AND PHASE NOISE}

The works of frequency estimation in the literature have tested the above algorithms only under additive Gaussian noise (AWGN), however, no test has been performed under phase noise (PN).

The main source of noise in electronic and communication systems is the thermal noise. This noise process (which is normally additive) is generated due to the random thermal agitation of free electrons as an electrical current passes through a conductor. This type of noise is white, i.e. it is composed of all frequencies. Another form of noise affecting communication systems is called phase noise [24]. This noise is created during the process of combination and recombination of charge carriers inside the molecular structure of the semiconductor. Hence, the sinusoidal signal with a fundamental frequency $f_{o}$ is disturbed by noise in the phase part, leading to a slight fluctuation in the instantaneous frequency. This is so because the instantaneous frequency $f(t)$ and phase $\varphi(t)$ are related by the instantaneous formula [4]:

$f(t)=\frac{1}{2 \pi} \frac{d \varphi(t)}{d t}$

In this work, we consider phase noise (PN) affecting the phase of a single-tone sinusoid as follows:

$x(t)=A \cdot \cos \left(\omega_{o} t+\emptyset_{o}+\rho(t)\right)+\epsilon(t)$

where $A$ is the signal amplitude, $\omega_{0}$ is angler frequency, $\left(\emptyset_{o}\right)$ Initial phase, $\rho(t)$ is the phase noise and $\epsilon(t)$ is the additive white Gaussian noise. This is just an extension to Equation (1) above. The above parameters are assumed to be unknown. We formulated PN as Gaussian noise added to the phase of the signal. This is the simplest model for phase noise.

\section{Simulation Results}

We simulated the above algorithms with signal model with AWGN and PN as per Equation (8) using MATLAB. The simulated signal has time length $L=70$ s, sampling interval $T_{s}=0.001 \mathrm{~s}, f_{s}=100 \mathrm{~Hz}$, and a number of samples $N=$ $\left[\frac{L}{T_{S}}\right]$. The signal amplitude is $A=1$ volt, $\omega_{\mathrm{o}}$ is angler frequency $\omega_{o}=2 \pi f_{o}$, where $f_{o}=23 \mathrm{~Hz}$. We modeled PN as zero-mean Gaussian noise. Monte Carlo simulations were performed with $M=100$ realizations. We used the quadratic frequency compensation as per Equation (4):

$u=K+d$; with $d=\left(u_{3}-u_{1}\right) /\left[2 *\left(2 * u_{2}-u_{1}-u_{3}\right)\right]$, and estimated frequency $F_{O}=\frac{u f_{S}}{N}$.

The signal-to-noise ratio (SNR) is still defined as before, i.e., using the AWGN power only. This is so because the phase noise power is affecting the phase only but not the amplitude of the signal.

Finally, we calculate the relative squared-error under each SNR and PN power as follows:

$$
e=\left|\left(\left(F_{o}-f_{o}\right) / f_{o}\right)\right|^{2}
$$

As for the frequency estimated by correlation, we do not calculate all the correlation coefficients of the signal to get the estimate, but only the 2 nd coefficient was considered. Note that we used Hilbert transformation (HT) to get the analytic signal $z(t)$ associated with the original signal $x(t)$ before estimation. This is to remove the negative part of the signal spectrum $X(f)$, where:

$z(t)=x(t)+j \cdot \mathcal{H}[x(t)]$

$\mathcal{H}[x(t)]=\left[\frac{1}{\pi t}\right] *_{t}[x(t)]$

noting that $*_{t}$ denotes time-convolution, and $\mathcal{H}$ denotes HT [25]. Hence:

$Z(f)=X(f)\left[1-j^{2} \cdot \operatorname{sgn}(f)\right]=X(f)[1+\operatorname{sgn}(f)]$

$\therefore \quad Z(f)=\left\{\begin{array}{cc}2 X(f) ; & f \geq 0 \\ 0 ; & f<0\end{array}\right\}$

Therefore, using HT will not affect the frequency estimation.

After estimation, we calculate relative squared-error for each SNR as follows:

$$
\mathbb{e}=\left|\frac{\left(\mathcal{F}_{o}-f_{o}\right)}{f_{o}}\right|^{2}
$$
(2).

Finally, we draw our results as shown in Figures (1) and

Note that taking more correlation coefficients (hence, more estimations for the frequency) will give more accurate results, but this is not recommended for real-time applications.

Figure (1) shows the estimated frequency versus SNR using interpolated FT peak and correlation methods for various powers of phase noise (PN). Numbers 1, 2, 3 correspond to PN powers of $-50,1,5 \mathrm{~dB}$, respectively. Note that FT hold in a high SNR less -30dB, as for to the correlation method holds to $-15 \mathrm{~dB}$, It is clear that PN does not affect CRB, as all curves converge to the same asymptote for large SNR. For all PN powers, FT peak outperforms correlation by almost $15 \mathrm{~dB}$. Also, it is clear that FT and correlation have the same CRB [as per Equation (2)], since both estimates have the same asymptote.

Figure (2) shows the frequency estimation mean-squared error (MSE) versus SNR using interpolated FT peak and correlation methods for various powers of phase noise (PN). Numbers 1, 2, 3 correspond to PN powers of $-50,1,5 \mathrm{~dB}$, respectively. It is clear that FT peak is more robust under very low SNR; however, it is more computationally expensive. This is not a surprise because correlation is highly dependent on phase.

\section{CONCLUSIONS}

We tested two popular frequency estimation algorithms, MLE through FFT and Correlation, using complex single-tone sinusoid affected by additive Gaussian and phase noise. Results of implementing these methods in MATLAB helped in comparing between them as follows:

- Fourier Transform (FT) approach is more efficient than the correlation approach (Corr) for frequency estimation. This is so because FT can work under low SNRs (as low as $-30 \mathrm{~dB}$ ), while the lowest SNR for the correlation method is $(-15 \mathrm{~dB})$, hence there is about ($15 \mathrm{~dB}$ ) difference between the two approaches. 
- FT outperforms Corr under phase noise, as it gives better $f_{0}$ estimation (lower error) at higher PN power values. This is so because Corr method is dependent on phase, so it will be more sensitive to phase noise.

- It is clear that PN does not affect CRB, as all error curves converge to the same asymptote for large SNR. Hence, both FT and Corr approaches have the same CRB.

- Despite the superiority of FT in frequency estimation as compared with Corr, the FT approach is computationally expensive. This so because FT requires the whole signal and estimates the frequency from the peak of FT, while in Corr approach we can take one correlation coefficient to estimate the frequency.

\section{REFERENCES}

[1] ZHANG Gang-bing, LIU Yu, XU Jia-jia, HU Guo-bing, "Frequency Estimation Based on Discrete Fourier Transform and Least Squares," IEEE International Conference on Wireless Communications \& Signal Processing (WCSP), 2009.

[2] Zahir M. Hussain and Boualem Boashash, "Multi-component IF estimation," Proceedings of the IEEE Signal Processing Workshop on Statistical Signal and array Processing (SSAP'2000), Pocono Manor, Pennsylvania, USA, pp. 559-563, 14-16 Aug. 2000.

[3] Zahir M. Hussain and Boualem Boashash, "Design of time-frequency distributions for amplitude and IF estimation of multicomponent signals," invited paper for the Statistical Time-Frequency Special Session in the Sixth International Symposium on Signal Processing and Its Applications (ISSPA'2001), vol. 1, pp. 339-342, Aug. 2001.

[4] Zahir M. Hussain and Boualem Boashash, "Adaptive instantaneous frequency estimation of multi-component FM signals using quadratic time-frequency distributions," IEEE Transactions on Signal Processing, vol. 50, no. 8, pp. 1866 -1876, August 2002.

[5] Yizheng Liao, Phase and Frequency Estimation: High-Accuracy and Low-Complexity Techniques, M.Sc. Thesis, Worcester Polytechnic Institute, 2011.

[6] E. Jacobsen, "On Local Interpolation of DFT Outputs," EF Data Corporation Report [Online, 1994]. Available: http://www.ericjacobsen.org/FTinterp.pdf.

[7] D. C. Rife, R. R. Boorstyn, "Single-Tone Parameter Estimation from Discrete-Time Observations," IEEE Trans. on Information Theory, v. 20, n. 5, 1974.

[8] B. G. Quinn, "Estimating Frequency by Interpolation Using Fourier Coefficients," IEEE Trans. Signal Processing, Vol. 42, no. 5, 1994.
[9] T. Grandke, "Interpolation Algorithms for Discrete Fourier Transforms of Weighted Signals," IEEE Trans. Instrumentation and Measurement, Vol. IM-32, no.7, 1983.

[10] B. Bischl, U. Ligges, C. Weihs, "Frequency Estimation by DFT Interpolation: A Comparison of Methods," Technical Report, Technische Universität Dortmund, 2009.

[11] V. Clarkson, "Efficient Single Frequency Estimators," International Symposium on Signal Processing and Its Applications (ISSPA), 1992.

[12] B. G. Quinn, "Estimation of Frequency, Amplitude, and Phase from the DFT of a Time Series," IEEE Trans. Signal Processing, Vol. 45, no. 3, 1997.

[13] Cui Yang, Gang Wei, and Fang-jiong Chen, "An Estimation-Range Extended Autocorrelation-Based Frequency Estimator," EURASIP Journal on Advances in Signal Processing, Volume 2009.

[14] L. B. Jackson and D.W. Tufts, "Frequency Estimation by linear prediction," IEEE International Conference on Acoustics, Speech and Signal Processing (ICASSP '78), USA, 1978.

[15] P. Händel, "Markov-Based Single-Tone Frequency Estimation," IEEE Trans. Circuits Syst. II, vol. 45, no. 1, 1998.

[16] G. W. Lank, I. S. Reed, and G. E. Pollon, "A Semi-Coherent Detection and Doppler Estimation Statistic," IEEE Trans. Aerosp. Electron. Syst., vol. AES-9, 1973.

[17] S. Kay, "A Fast and Accurate Single Frequency Estimator," IEEE Trans. Acoustics, Speech, and Signal Processing, no. 12, 1989.

[18] E. Jacobsen and P. J. Kootsookos, "Fast, Accurate Frequency Estimators," IEEE Signal Processing Magazine, May 2007.

[19] P. Händel, A. Eriksson, and T. Wigren, "Performance Analysis of a Correlation Based Single Tone Frequency Estimator," Signal Processing, vol. 44, no. 2, no. 6, 1995.

[20] M. P. Fitz, "Further Results in the Fast Estimation of a Single Frequency," IEEE Trans. Coinniun.1994.

[21] D. W. Tufts and P. D. Fiore, "Simple, Effective Estimation of Frequency Based on Pony's Method," IEEE Int. Conf. Acoust., Speech, Signal Process., vol. 5, 1996.

[22] P. Händel, B. Völcker, and B. Göransson, "Analysis of a Simple, Effective Frequency Estimator Based on Prony's Method," IEEE Signal Process. Workshop Stat. Signal Array Process., Sept. 1998.

[23] B. Völcker, P. Händel, "Frequency Estimation From Proper Sets of Correlations," IEEE Trans. Signal Process., vol. 50, no. 4, April 2002.

[24] R. Corvaja and S. Pupolin, "Phase noise effects in QAM systems," IEEE Int. Symp. on Personal, Indoor and Mobile Radio Commun., vol. 2, no. 2, Sep. 1997.

[25] Zahir M. Hussain and Boualem Boashash, "Hilbert transformer and time-delay: statistical comparison in the presence of Gaussian noise," IEEE Transactions on Signal Processing, vol. 50, no. 3, pp. 501-508, March 2002 


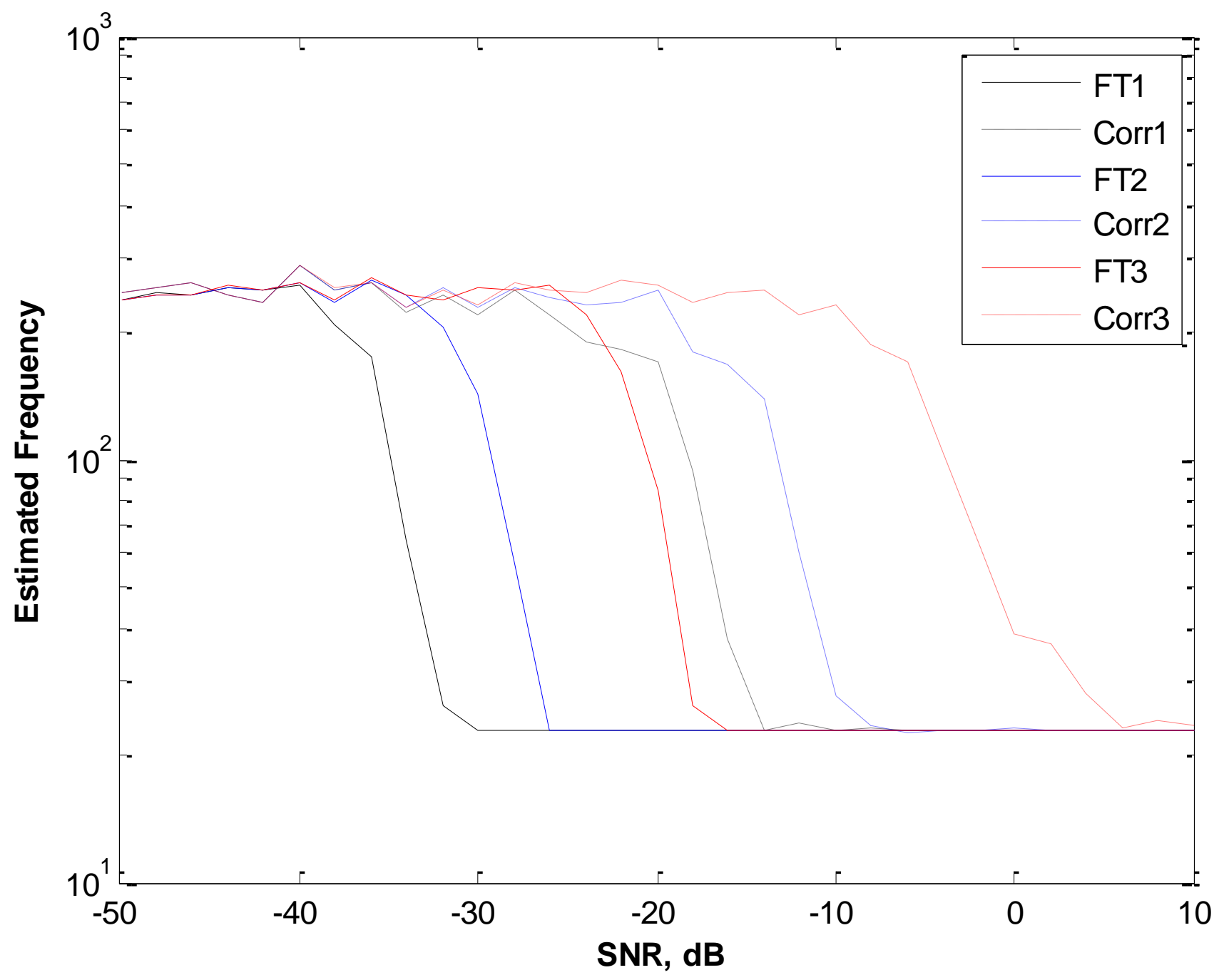

Fig. 1. Estimated frequency versus SNR using interpolated FT peak and correlation methods for various powers of phase noise (PN). Numbers 1, 2, 3 correspond to PN powers of -50 (no noise), $1,5 \mathrm{~dB}$, respectively. Note that SNR is only considered for AWGN. 


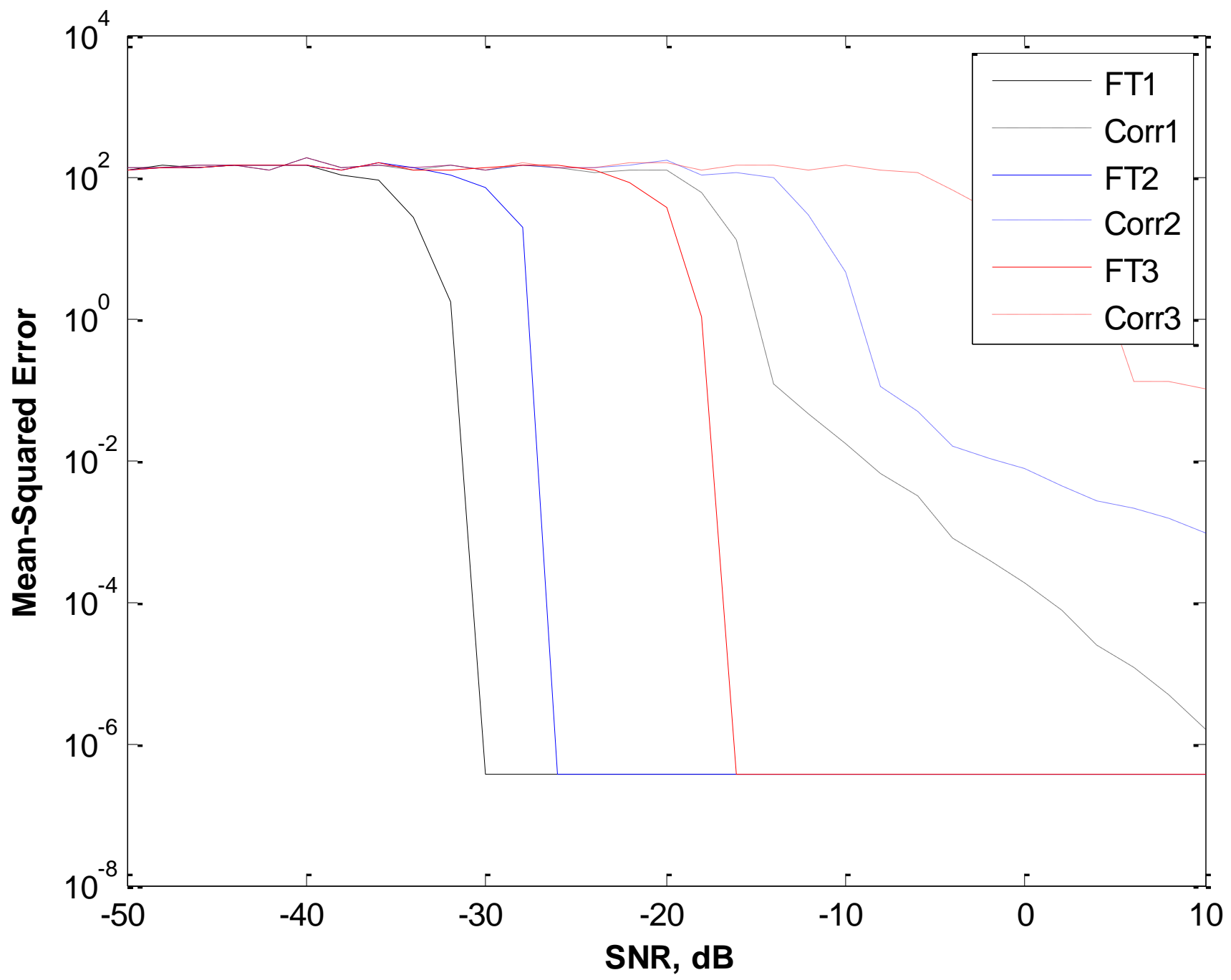

Fig. 2. Frequency estimation mean-squared error (MSE) versus SNR using interpolated FT peak and correlation methods for various powers of phase noise (PN). Numbers 1, 2, 3 correspond to PN powers of $-50,1,5 \mathrm{~dB}$, respectively. It is clear that FT peak is more robust under very low SNR. 